\title{
Design and Use of Wafer Level Fluidic Packaging for Surface Acoustic Wave Sensors
}

\author{
J.-M. Friedt and L. El Fissi \\ SENSeOR \\ 399 route des Crêtes, \\ Sophia Antipolis, France
}

\author{
F. Chérioux, B. Guichardaz, V. Blondeau-Patissier, S. Ballandras \\ FEMTO-ST/LPMO \\ 32, avenue de l'Observatoire \\ 25044 Besançon, France
}

\begin{abstract}
We demonstrate the propagation of a Love mode acoustic wave in a thin SU8 epoxy layer coating a AT-cut quartz substrate: the resulting insertion losses are observed in the $\mathbf{- 3 5}$ to $-40 \mathrm{~dB}$ range. We demonstrate the packaging of this device in a SU8 microfluidic system preventing liquids from covering the interdigitated transducer while defining the chemical reaction area in the region between the interdigitated transducer. We demonstrate the ability to chemically functionnalize the SU8 layer with amine-ended groups. Finally, a time-resolved adsorption of small (600 daltons) organometallic molecules is presented for illustrating the detection sensitivity and selectivity of the resulting sensor working with analytes in acqueous solutions.
\end{abstract}

\section{INTRODUCTION}

Acoustic sensors are part of the wider class of direct detection sensors in which no preliminary preparation of the sample to be analyzed is necessary. Direction detection sensors used for detecting biological species - biosensensors -must be able to work with samples provided in liquid medium. Optical sensors such as Surface Plasmon Resonance or acoustic sensors such as Quartz Crystal Resonators (also called Microbalance) are compatible with use with liquid samples by use of appropriate fluidics handling packagings. We are here interested in Surface Acoustic Wave sensors which provide unique capabilities for the detection of low levels of analytes in liquid samples: no electrodes or structures in the sensing area, only one side of the quartz substrate is patterned with electrodes, high sensitivity, compatibility with liquids when an appropriate (shear) acoustic wave is selected, high integration allowing differential measurement for the automatic compensation of environmental disturbances (temperature, acceleration, ...).

The main issue with the use of Surface Acoustic Sensors [1]-[4] - especially in a commercial objective - is the development of a reliable and reproducible fluidic system [5] meeting the following main requirements:

- liquid tight insulation of the interdigitated transducers (IDTs) classically used for generating surface acoustic waves on a piezoelectric substrate. Bringing high permittivity solvents over the IDTs will capacitively short circuit the transducers leading to strong electrical losses and hence poor acoustic response, usually by bringing the acoustic signal below the electromagnetic background level,
- low acoustic losses since the fluidic system must necessarily cover the acoustic path. Two strategies can help meet this requirement: deposit a thin wall on the acoustic path in order to reduce the interaction between the propagating acoustic wave and the mass loading of the wall on the acoustic path ; and use of a material with an acoustic impedance as different as possible than that of the guiding path in the case of a guided Love mode acoustic wave sensor. The second strategy ensures that the acoustic wave stays confined in the waveguide created by the low velocity waveguide deposited on top of the piezoelectric substrate,

- chemically inert to biological samples while resistant to solvents used during the surface functionalization and cleaning steps (organic solvents and acids),

- reproducible fabrication at the wafer scale level in order to meet economic constraints of an industrial fabrication process.

We have selected a delay line configuration for ease of use, providing a wide band acoustic signal probed in an open-loop configuration by a lab-made dedicated network analyzer and significantly, a large sensing area compatible with a human operator (Fig. 1).

Based on previous experiments in which pushing a PDMS liquid cell on the acoustic sensor led to irreproducible acoustic responses varying over time and experiments and leading to poor liquid tightness, we focused on the patterning of fluidic caps on the acoustic device using cleanroom patterning technology processes [6]. We assume here that by accurately patterning the same obstacles on the acoustic path using microelectronic techniques, we should be able to provide constant and acceptable disturbances for the further use of the sensors in a liquid environment. Once the obstacles are properly positioned on the acoustic device and provide sufficient insulation, classical fluidic techniques (PDMS, gluing, Viton seals, Teflon cells ...) can be pressed on top of these walls without affecting the acoustic wave which is only affected by it environment up to a few wavelength around (in our case the wavelength $\lambda=40 \mu \mathrm{m}$ was selected as a compromise between manufacturing capabilities and high frequency leading to high mass sensitivity).

Patterning microfluidic devices on the Love mode guiding layer requires good adhesion between the materiel the 


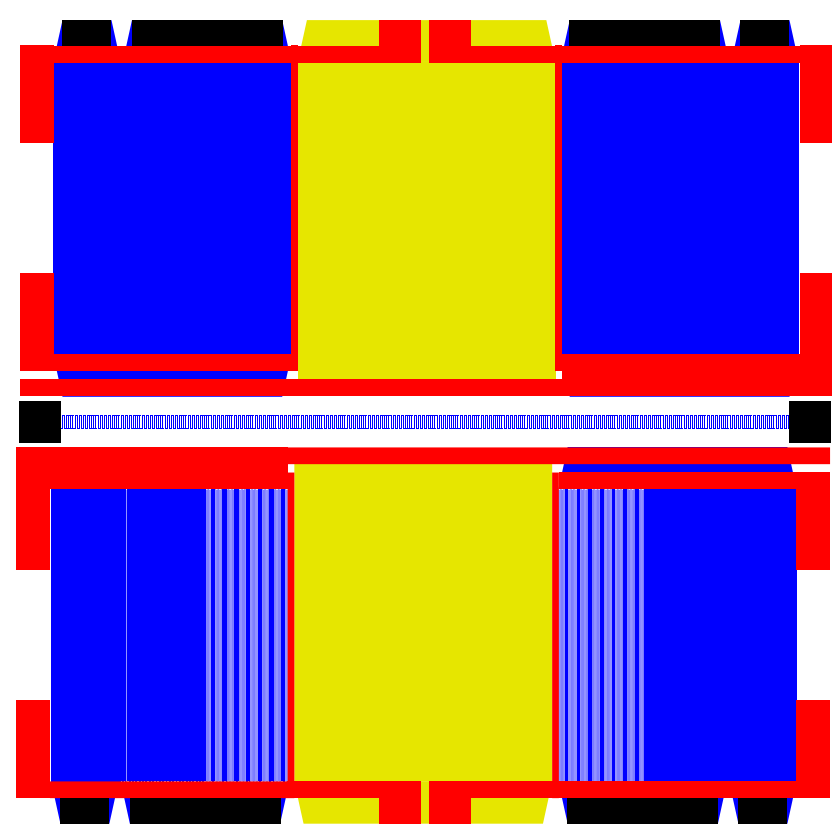

Fig. 1. General layout of the dual delay line device developed: blue represents the $A l$ IDTs $(\lambda=40 \mu \mathrm{m}$ with two fingers/electrical period (i.e. $5 \mu \mathrm{m}$ wide finger pairs separated by $5 \mu \mathrm{m}$ wide gaps), yellow is the sensing area and red represented one possible layout for a fluidic system preventing the liquid coating the sensing area from reaching the IDTs. The pattern between the two delay lines is a thermistor.

microfluidics is made of and the guiding layer. In order to improve adhesion with respect to the classical $\mathrm{SiO}_{2}$ guiding layer, we have here focused on selecting a proper guiding layer: we have thus focused on propagating a Love wave in a thin SU8 guiding layer. We are indeed aware that even with high aspect ratio structures, SU8 patterns will adhere well to a thin SU8 layer. Furthermore, propagating the Love wave in a polymer layer rather than an inorganic layer deposited in a vacuum chamber greatly shortens the global fabrication time of the biosensor. The main drawback is the increased acoustic losses ofter associated with acoustic wave propagation in polymers: the still epoxy layer created by SU8 is expected to yield acceptable results.

We here demonstrate the propagation of a Love mode acoustic wave in a $1 \mu \mathrm{m}$ thick SU8 layer with acceptable acoustic losses, and illustrate the use of the acoustic sensor covered by a thick SU8 microfluidic pattern for detecting organometallic complexes following amine-ended functionalization of the surface. This functionalization was chosen as relevant to further biochemical functionalizations, while it demonstrates the ability of the full device to withstand organic solvents during the functionalization process. The organometallic complexes were chosen since their affinity to amine groups was previously demonstrated [7], [8] and their specific XPS response provides an easy means of independently demonstrating the functionalization step.

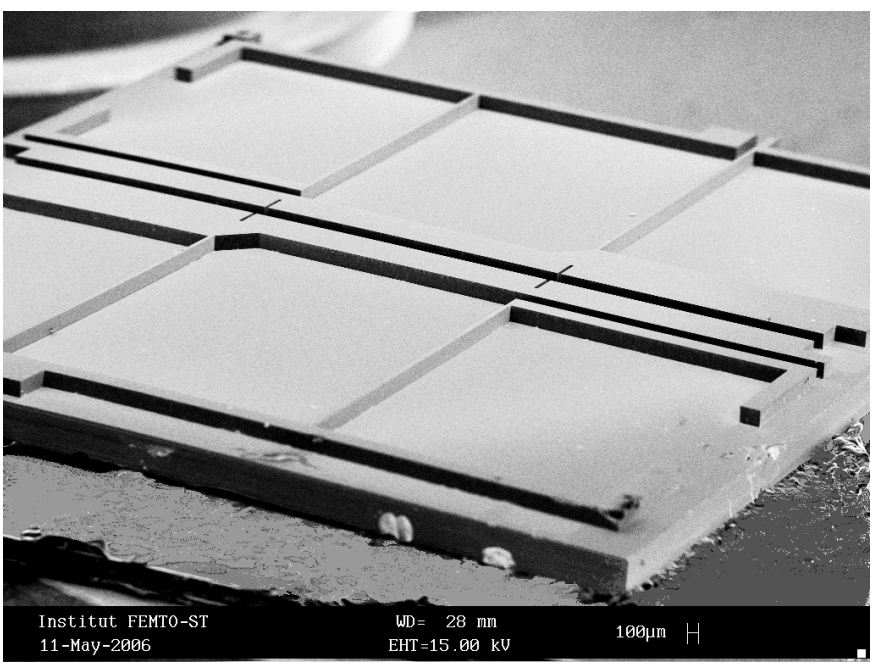

Fig. 2. SEM image of the SAW device coated with a patterned thick SU8 layer: the critical elements are the two walls locate on the acoustic path which should be as thin as possible (here $40 \mu \mathrm{m}=\lambda$ ) and as high as possible (150 $\mu \mathrm{m}$ $\geq 3 \lambda$ ) in order to minimize acoustic losses while still preventing the liquid from leaking onto the IDTs.

\section{LOVE MODE ACOUSTIC WAVE SENSOR}

Split fingers IDTs made of $200 \mathrm{~nm}$ thick evaporated $\mathrm{Al}$ were patterned on AT-cut quartz to generate an SSBW acoustic wave. This wave is converted to a guided Love mode by a $1 \mu \mathrm{m}$ SU8 spin-coated epoxy layer. The resulting sensor is thus made of 50 pairs of IDTs separated by a cavity $3.2 \mathrm{~mm}$ long and $3.5 \mathrm{~mm}$ wide. The acoustic aperture is $3.5 \mathrm{~mm}$. The SU8 photoresist used here as guiding layer is patterned for opening the bonding pads.

The insertion loss after SU8 polymerization is observed to be $-39 \mathrm{~dB}$ (Fig. 3). Although this value is greater than that usually observed for inorganic layers (typically in the -25 to $30 \mathrm{~dB}$ range for silicon dioxyde), the expected high sensitivity expected from the slow acoustic velocity in the guiding layer compensates the difficulties in using sensors with such high insertion losses.

Having selected an epoxy-based guiding layer for producing a sensivite acoustic devices and strong fluidic packaging (Fig. 2 ), the remaining issue is the ability to use surface chemistry functionalization for binding receptors to the analyte to be detected.

\section{MICROFLUIDIC PACKAGING}

We have used a two steps method to fabricate the liquid cell:

- The first step is achieved at the wafer level. It consists in the patterning of the SU-8 walls.

The resulting sensor is glued on a FR4 epoxy substrate with electrical The SU-8 walls with a thickness of about $120 \mu \mathrm{m}$ were obtained by using the following recipe. Photosensitive SU-8 type 2075 was spin coated with an acceleration of $100 \mathrm{RPM} / \mathrm{s}$ to reach $500 \mathrm{RPM}$ that was held for 10 seconds, and followed by an acceleration of 

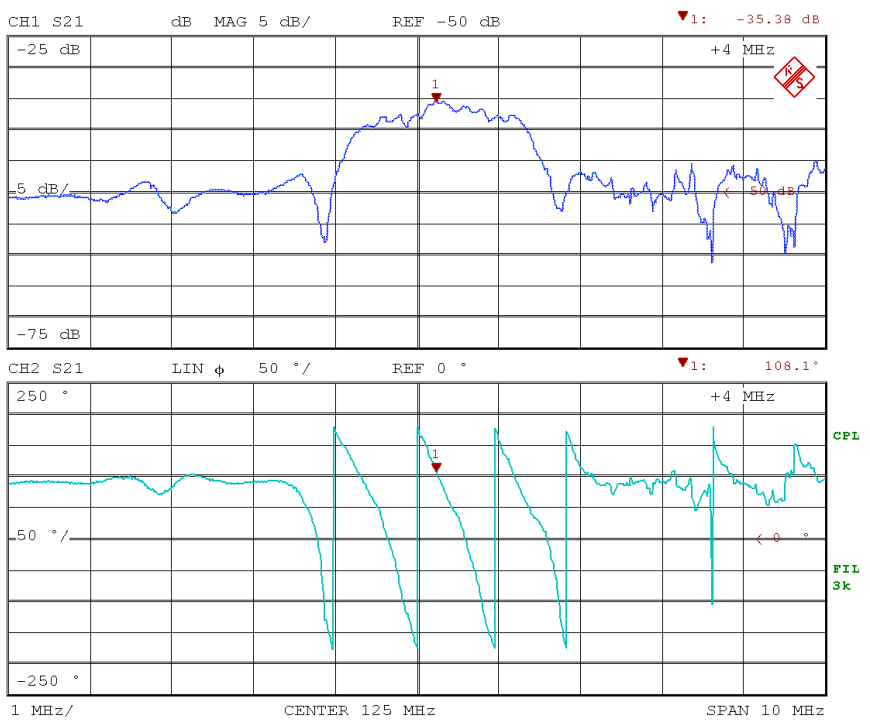

Date: 4.MAT.06 10:20:27

Fig. 3. Typical $S_{21}$ transfer function of a $40 \mu \mathrm{m}$ wavelength Love mode acoustic wave propagating in a $1.2 \mu \mathrm{m}$ thick SU8 layer patterned with $150 \mu \mathrm{m}$ high, $40 \mu \mathrm{m}$ wide structures on the acoustic path (see Fig. 2).

(a)

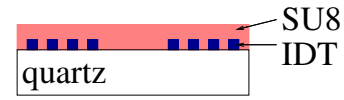

(b)

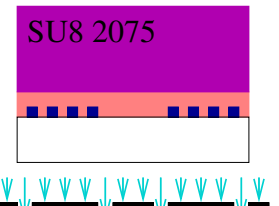

(c)

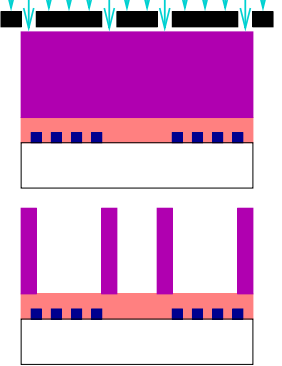

Fig. 4. SU8 walls fabrication steps. (a) Love wave delay line with SU8 guiding layer ; (b) spin coating of SU8 2075 and soft-bake ; (c) soft contact lithography and post-bake ; development of SU8.

$300 \mathrm{RPM} / \mathrm{s}$ to reach $1400 \mathrm{RPM}$ that was held during 25 seconds. After spinning, the resist was baked in an oven for 5 hours at $75^{\circ} \mathrm{C}$ after a slow temperature ramp up in order to avoid stresses cracks in the epoxy layer. The SU-8 patterns were obtained by soft contact lithography. The exposure was followed by a 30 minute bake at $75^{\circ} \mathrm{C}$ in an oven after a slow temperature ramp up to finish the reticulation of the SU8. The unexposed epoxy was then dissolved in propylene glycol monomethyl ether acetate (PGMEA). Fig. 4 summarizes this fabrication process. Following that step, the wafer was diced to release each sensor with the patterned SU8 walls.
- For the second step, glass pieces of $5 \mathrm{~mm}$ by $5 \mathrm{~mm}$ and $350 \mu \mathrm{m}$ thick were placed and glued above the SU8 structures. An epoxy glue (Epotek H70) was manually dispensed above the walls and followed by the placement of the glass.

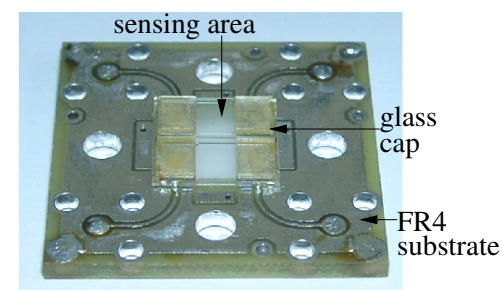

Fig. 5. Packages SAW sensor cartridge

The resulting sensor is glued on a FR4 epoxy substrate with electrical connections being performed by conducting silver loaded epoxy between the bonding pads and the copper tracks on the circuit Fig. 5.

\section{Chemical FUnCTIONALiZATION AND DETECTION REACTIONS}

We have focused on demonstrating our ability to use a silane chemistry to bind amine-ended molecules to the SU8 surface. In order to demonstrate the successful functionalization step, we have furthermore focused on monitoring the binding of organometallic complexes to this surface: the metal atoms ( $R u$ and $R h$ in our case, included in dinuclear dichloro complexes $\left[R u\left(\eta^{6}-C_{6} H_{6}\right) C l_{2}\right]_{2}$ and $\left[R h\left(\eta^{5}-C_{5} M e_{5}\right) C l_{2}\right]_{2}$ respectively, synthesized as described in [9]-[11]) provide unambiguous signatures during XPS characterization. Hence the focus of the time resolved adsorption monitoring in an open loop (network analyzer monitoring at fixed frequency the evolution of the insertion loss and phase evolution during the chemical reaction cycles) is first to use the acoustic sensor for detecting an organomatallic species dissolved in water, and secondly to provide a marker of the functionalization of the SU8 guiding layer.

Having functionalized the SU8 surface by first exposing the surface to a UV-ozone treatement for 30 minutes followed by an overnight stay in 3-(dimethoxymethylsilyl)-1-propylamine dissolved in toluene (Fig. 6, top) we have run the adsorption experiment as follows (Fig. 6, bottom):

1) define the initial baseline in DI water by multiple injection and removal of DI water for making sure that the baseline is stable to fluidic manipulation,

2) inject the solution containing the organometallic complex and water for a stable signal to be reached again.

3) have reached a stable signal again, the liquid cell is rinsed with DI water multiple times and a new baseline in DI water is observed. The phase and insertion loss between the initial and current baselines is associated with molucules bound to the surface.

4) in order to demonstrate full saturation and irreversible binding of the organometallic complexes to the surface, 


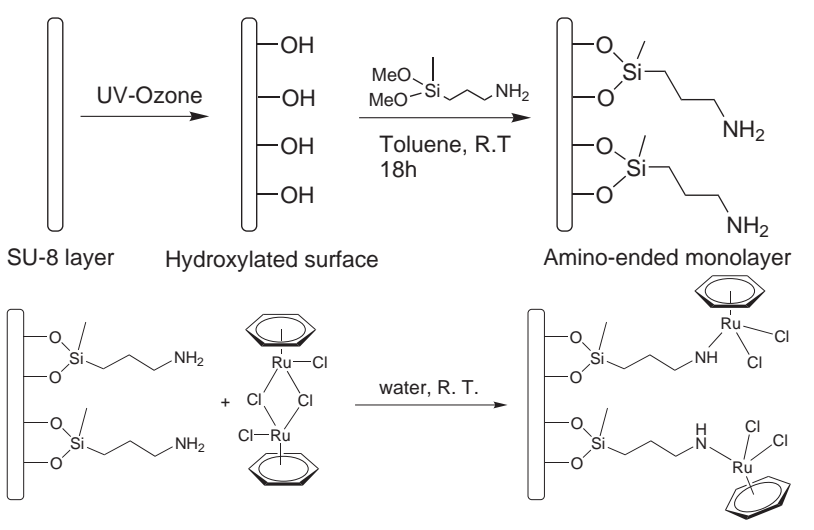

Fig. 6. Top: SU8 functionalization steps. Bottom: organometallic complex binding to the functionalized surface.

we reiterate the last 2 steps and verify that the signal level of the device coated with DI water stays constant.

The control experiment of submitting the bare - nonfunctionalized - SU8 surface to the organometallic containing solution led to no visible acoustic signal modification. Hence, the measured signal is attributed to the covalent binding of the complexes to the amine groups, which proves the SU8 functionalization.

\section{RESULTS AND DISCUSSION}

The most unexpected result of these adsorption experiments is that the most significant signal arises on the magnitude signal. Thin film adsorption on acoustic sensors are usually associated with a decrease in acoustic velocoty which is mostly observed on the phase signal in an openloop monitoring configuration. Here the SU8 surface is directly coated with the organometallic complex without shielding by a grounded conducting metallic layer on the sensing area. Hence, the acoustic signal is not only sensitive to gravimetric effects hence the common denomination of microbalance of these acoustic devices - but also to permittivity changes leading to modifications of the electric field distribution close to the surface. We attribute the predominant response in terms of insertion loss rather than phase shift to be due to such a permittivity modification rather than gravimetric effect. This results emphasises the difficulty of interpreting measurements performed with acoustic sensors whose sensing area is not coated by a shielding conducting layer.

Beyond the quantitative interpretation of these signals, we here demonstrate the sensitivity of such sensors since a significant signal results from a small molecule monolayer: the molecular weight of the organometallic compounds investigated here is in the 600 daltons range.

Wafer scale sealing of cavities yields new challenges: the surface on top of the patterned walls must be flat enough and of homogeneous thickness to allow further sealing by a top-wafer. Most wall-patterning techniques (thick photoresist coating, metal electrodeposition [12], [13], wafer gluing) has shown the challenge of obtaining a flat surface on which a fluidic capping could be glued.
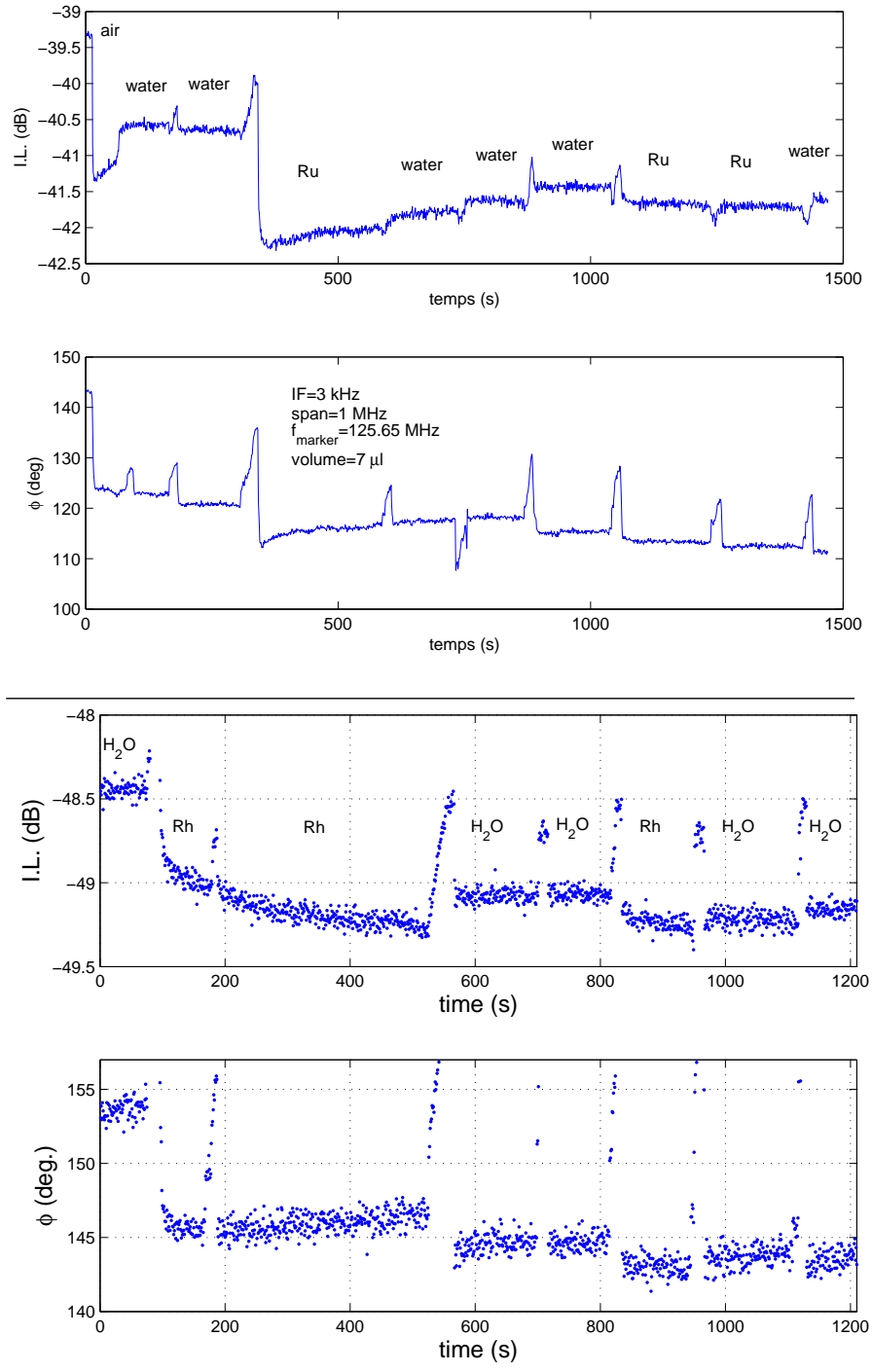

Fig. 7. Time resolved organometallic complex adsorption to the amineended functionalized SU8 guiding layer detected by phase and magnitude measurement of a $40 \mathrm{mum}$ wavelength Love mode acoustic wave. Top : magnitude and phase measurements monitored by a network analyzer at fixed frequency during $R u$ organometallic complex adsorption. Bottom : magnitude and phase measurements monitored by a network analyzer at fixed frequency during $R h$ organometallic complex adsorption.

\section{CONCLUSION AND PERSPECTIVES}

We have demonstrated here the ability to propagate a Love mode acoustic wave on an organic epoxy layer made of SU8 photoresist. The selected material for guiding the acoustic wave provides a large acoustic velocity between the piezolectric substrate (quartz) and the guiding layer (SU8) yielding improved sensitivity with respect to inorganic (faster) guiding layers. Furthemore, such an organic layer provies an appropriate surface for further patterning a microfluidic system using cleanroom wafer scale processing techniques. We have thus demonstrated a packaging method preventing leakage of the solvent containing the molecule under investigation during the chemical functionalization step in an organic solvent and further time-resolved measurements during the adsorption 
step. The observed acoustic signal - confirmed with XPS post-analysis and control experiments - yields unambiguous confirmations of the ability to chemically functionalize SU8 surface with amine-ended groups.

\section{REFERENCES}

[1] E. Gizeli, Biomolecular Sensors, E. Gizeli and C. Lowe, Eds. Taylor \& Francis: London, 2002.

[2] A. Wang, J. Cheeke, and C. Jen, "Sensitivity analysis for love mode acoustic gravimetric sensors," Appl. Phys. Lett., vol. 64, no. 22, pp. 2940-2942, 1994.

[3] B. Jakoby and M. Vellekoop, "Viscosity sensing using a love-wave device," Sensors and Actuators A, vol. 68, pp. 275-281, 1998.

[4] J. Du, G. Harding, J. Ogilvy, P. Dencher, and M. Lake, "A study of lovewave acoustic sensors," Sensors and Actuators A, vol. 56, pp. 211-219, 1996.

[5] K. Länge, G. Blaess, A. Voigt, R. Götzen, and M. Rapp, "Integration of a surface acoustic wave biosensor in a microfluidic polymer chip," Biosensors and Bioelectronics, vol. 22, pp. 227-232, 2006.

[6] L. Francis, J.-M. Friedt, C. Bartic, and A. Campitelli, "A su8 liquid cell for surface acoustic waves biosensors," 2004.

[7] F. Chérioux, C. M. Thomas, B. Therrien, and G. Süss-Fink, "Dendritic systems based on dinuclear rhodium or ruthenium units generating peripheric catalytic sites," Chem. Eur. J., vol. 8, pp. 4377-4382, 2002.

[8] M. J.-L. Tschan, L. Karmazin-Brelot, F. Chérioux, and G. SüssFink, "Reactivity of the unsaturated complex $\left[\left(C_{6} M e_{6}\right)_{2} R u_{2}(H)_{3}\right]^{+}$ towards phosphines: Synthesis, molecular structure of the dinuclear cations $\left[\left(C_{6} M e_{6}\right)_{2} R u_{2}\left(P R_{2}\right)(H)_{2}\right]^{+}$, and characterisation of a pc bond activation intermediate $\left[\left(C_{6} M e_{6}\right)_{2} R u_{2}\left(P P h_{2}\right)(P h)(H)\right]^{+}$," Organometallics, vol. 24, pp. 1974-1981, 2005.

[9] R. Zelonka and M. Baird, "Benzene complexes of ruthenium(II)," Can. J. Chem., vol. 50, pp. 3063-3068, 1972.

[10] J. Wang, K. Moseley, and P. Maitlis, "Pentamethylcyclopentadienylrhodium and -iridium halides. i. synthesis and properties," J. Am. Chem. Soc., pp. 5970-5977, 1969.

[11] M. Bennett and A. Smith, "Arene ruthenium(II) complexes formed by dehydrogenation of cyclohexadienes with ruthenium(III) trichloride," $J$. Chem. Soc, Dalton Trans., pp. 233-241, 1974.

[12] L. Robert, S. Basrour, M. Wery, and F. Sittler, "The morphology and electrochemical behavior of the electrodeposition of nickel onto metallized silicons," Plating and Surface Finishing, vol. 87, no. 5, pp. 153-159, 2000.

[13] W. Daniau, S. Ballandras, P. Berçot, and D. Hauden, "Metallic microdevices fabricated by deep etch uv lithography," Materials Science and Engineering, vol. A160, pp. L5-L8, 1993. 